\title{
Planck and Electroweak Scales Emerging from Weyl Conformal Gravity
}

\author{
Ichiro Oda* \\ Department of Physics, Faculty of Science, University of the Ryukyus, \\ Nishihara, Okinawa 903-0213, Japan \\ E-mail: iodadsci.u-ryukyu.ac.jp
}

\begin{abstract}
We show that the Planck mass scale can be generated from conformal gravity in the Weyl conformal geometry via the Coleman-Weinberg mechanism of dimensional transmutation where quantum corrections stemming from the gravitational field and the Weyl gauge field trigger the symmetry breakdown of a local Weyl symmetry. It is also shown that the vacuum expectation value of a scalar field is transmitted to a sector of the standard model through a potential involving the scale invariant part and the contribution from the Coleman-Weinberg mechanism, thereby generating the electroweak scale.
\end{abstract}

Corfu Summer Institute 2018 "School and Workshops on Elementary Particle Physics and Gravity" (CORFU2018)

31 August - 28 September, 2018

Corfu, Greece

${ }^{*}$ Speaker. 


\section{Introduction}

One of the most important problems in modern particle physics is to understand the origin of different mass scales existing in nature. For instance, the electroweak mass scale $M_{E W}$ is generated via the Higgs mechanism where the Higgs field takes a vacuum expectation value around $100 \mathrm{GeV}$, while the QCD mass scale $\Lambda_{Q C D}$, which accounts for the masses of the nucleons and thus most of the visible mass in the universe (about $98 \%$ of the whole visible mass), is an example of the mass generation by dimensional transmutation.

In order to understand the origin of various mass scales, it is natural to start with a theory which has no intrinsic mass scales and consider how the mass scales are generated from a massless world via a certain mechanism. It is well known that there is a local or global scale symmetry in such a theory without intrinsic mass scales. However, as stressed in [四, if we couple the gravitational interaction to a theory, only local symmetries in general make sense since no-hair theorem []] of quantum black holes suggests that global additive conservation laws such as baryon and lepton number conservation cannot hold in any consistent quantum gravity theory. Indeed, in string theory, we never get any additive conservation laws and at least in known string vacua, the additive global symmetries turn out to be either gauge symmetries or explicitly violated. By contrast, gauge symmetries such as U(1) electric charge conservation law cause no trouble for black hole physics. Thus, in the gravitational theory, the global symmetries should be promoted to the local gauge symmetries except some unbroken global symmetries associated with boundary or topology of four-dimensional manifolds.

In this article, we wish to construct a gravitational theory with the standard model (SM) where the local scale symmetry plays a critical role, and then examine whether the Planck and the electroweak scales are generated or not. To this aim, we will embed the SM and general relativity (GR) in a larger theory which exhibits a local scale symmetry, and show that the Planck scale arises by the Coleman-Weinberg mechanism via dimensional transmutation [3] and it is then transmitted to the SM through a scale invariant potential, thereby generating the electroweak scale.

A natural and general framework where a scale symmetry is implemented as a local gauge symmetry is given by a generalization of the Riemann geometry, which is known as the Weyl geometry [四]. ${ }^{1}$ The Weyl geometry is defined as a geometry with a real symmetric metric tensor and a symmetric connection given by Eq. (2.5) as seen shortly. It is therefore expected that when the Weyl gauge field $S_{\mu}$ is vanishing, the Weyl geometry becomes equivalent to the Riemann geometry. However, it turns out that if $S_{\mu}$ is a gradient, i.e., pure gauge, the Weyl geometry precisely reduces to the Riemann one. In the Weyl geometry, parallel displacement of a vector field changes its length so that the very notion of length becomes path-dependent. For instance, one can envisage a space traveller, who travels to a distant star and then returns to the earth, being surprised to know not only that people in the earth have aged much rather than him as predicted by GR in the Riemann geometry but also that the clock on the rocket runs at a different rate from that in the earth as understood by Weyl conformal gravity in the Weyl geometry, what we call, "the second clock problem" [目]. Based on this very striking geometry, Weyl has succeeded in geometrizing the electromagnetic theory in the space-time geometry.

\footnotetext{
${ }^{1}$ See Ref. []] for historical review on the Weyl geometry.
} 
Since we have already applied the idea of generating the Planck scale from the ColemanWeinberg mechanism to locally scale invariant gravitational theories with the SM in the Riemann geometry $[\mathbb{W}, \mathbb{\square}]$, in this article we will deal with a locally scale invariant theory in the Weyl geometry and show that this is indeed the case even in this more general geometry. The detail will be reported in a separate publication in the near future.

\section{Weyl conformal geometry}

We start with a brief review on the basic concepts and definitions of the Weyl conformal geometry. ${ }^{2}$ We adopt the conventions and notation for the Riemann tensors and the metric signature in the Wald's textbook [ए]].

In the Weyl geometry, the Weyl transformation, which is the sum of the local conformal transformation for a generic field $\Phi(x)$ and the Weyl gauge transformation for a Weyl gauge field $S_{\mu}(x)$, is defined as

$$
\Phi(x) \rightarrow \Phi^{\prime}(x)=e^{w \Lambda(x)} \Phi(x), \quad S_{\mu}(x) \rightarrow S_{\mu}^{\prime}(x)=S_{\mu}(x)-\frac{1}{f} \partial_{\mu} \Lambda(x),
$$

where $w$ is the Weyl weight, $f$ is the coupling constant for the non-compact abelian gauge group, and $\Lambda(x)$ is the local parameter for the conformal transformation. Writing out the conformal transformation for various fields explicitly,

$$
\begin{aligned}
& g_{\mu v}(x) \rightarrow g_{\mu \nu}^{\prime}(x)=e^{2 \Lambda(x)} g_{\mu v}(x), \quad \phi(x) \rightarrow \phi^{\prime}(x)=e^{-\Lambda(x)} \phi(x), \\
& \psi(x) \rightarrow \psi^{\prime}(x)=e^{-\frac{3}{2} \Lambda(x)} \psi(x), \quad A_{\mu}(x) \rightarrow A_{\mu}^{\prime}(x)=A_{\mu}(x),
\end{aligned}
$$

where $g_{\mu v}(x), \phi(x), \psi(x)$ and $A_{\mu}(x)$ are the metric tensor, scalar, spinor, and conventional gauge fields, respectively. Here it is convenient to define a Weyl covariant derivative $D_{\mu}$ for a generic field $\Phi(x)$ with the Weyl weight $w$ as

$$
D_{\mu} \Phi \equiv \partial_{\mu} \Phi+w f S_{\mu} \Phi
$$

which transforms covariantly under the Weyl transformation:

$$
D_{\mu} \Phi \rightarrow\left(D_{\mu} \Phi\right)^{\prime}=e^{w \Lambda(x)} D_{\mu} \Phi .
$$

As mentioned above, the Weyl geometry is defined as a geometry with a real symmetric metric tensor $g_{\mu \nu}\left(=g_{v \mu}\right)$ and a symmetric connection $\tilde{\Gamma}_{\mu \nu}^{\lambda}\left(=\tilde{\Gamma}_{v \mu}^{\lambda}\right)$ which is defined as

$$
\begin{aligned}
\tilde{\Gamma}_{\mu v}^{\lambda} & =\frac{1}{2} g^{\lambda \rho}\left(D_{\mu} g_{v \rho}+D_{v} g_{\mu \rho}-D_{\rho} g_{\mu v}\right) \\
& =\Gamma_{\mu v}^{\lambda}+f\left(S_{\mu} \delta_{v}^{\lambda}+S_{v} \delta_{\mu}^{\lambda}-S^{\lambda} g_{\mu v}\right),
\end{aligned}
$$

where

$$
\Gamma_{\mu v}^{\lambda} \equiv \frac{1}{2} g^{\lambda \rho}\left(\partial_{\mu} g_{v \rho}+\partial_{v} g_{\mu \rho}-\partial_{\rho} g_{\mu v}\right),
$$

\footnotetext{
${ }^{2}$ See also Refs. [四, 四, 四] for a concise introduction of the Weyl geometry.
} 
is the affine connection in the Riemann geometry. The most important difference between the Riemann geometry and the Weyl one is that $\nabla_{\lambda} g_{\mu \nu}=0$ (the metric condition) in the Riemann geometry, while in the Weyl geometry

$$
\tilde{\nabla}_{\lambda} g_{\mu v} \equiv \partial_{\lambda} g_{\mu v}-\tilde{\Gamma}_{\lambda \mu}^{\rho} g_{\rho v}-\tilde{\Gamma}_{\lambda v}^{\rho} g_{\mu \rho}=-2 f S_{\lambda} g_{\mu v}
$$

Let us recall that the metric condition implies that length and angle are preserved under parallel transport whereas Eq. (‥]) does that only angle, but not length, is preserved by the Weyl connection.

Now using the Weyl connection $\tilde{\Gamma}_{\mu \nu}^{\lambda}$ one can construct a conformally invariant curvature tensor:

$$
\begin{aligned}
\tilde{R}_{\mu v \rho}^{\sigma} & \equiv \partial_{v} \tilde{\Gamma}_{\mu \rho}^{\sigma}-\partial_{\mu} \tilde{\Gamma}_{v \rho}^{\sigma}+\tilde{\Gamma}_{\mu \rho}^{\alpha} \tilde{\Gamma}_{\alpha v}^{\sigma}-\tilde{\Gamma}_{v \rho}^{\alpha} \tilde{\Gamma}_{\alpha \mu}^{\sigma} \\
& =R_{\mu v \rho}{ }^{\sigma}+f\left(\delta_{[\mu}^{\sigma} \nabla_{v]} S_{\rho}-\delta_{\rho}^{\sigma} \nabla_{[\mu} S_{v]}-g_{\rho[\mu} \nabla_{v]} S^{\sigma}\right) \\
& +f^{2}\left(S_{[\mu} \delta_{v]}^{\sigma} S_{\rho}-S_{[\mu} g_{v] \rho} S^{\sigma}+\delta_{[\mu}^{\sigma} g_{v] \rho} S_{\alpha} S^{\alpha}\right)
\end{aligned}
$$

where $R_{\mu v \rho} \sigma$ is the curvature tensor in the Riemann geometry, and we have defined the antisymmetrization by the square bracket, e.g., $A_{[\mu} B_{v]} \equiv A_{\mu} B_{v}-A_{v} B_{\mu}$. Then, it is straightforward to prove the following identities:

$$
\tilde{R}_{\mu v \rho}{ }^{\sigma}=-\tilde{R}_{v \mu \rho}{ }^{\sigma}, \quad \tilde{R}_{[\mu v \rho]}{ }^{\sigma}=0, \quad \tilde{\nabla}_{[\lambda} \tilde{R}_{\mu v] \rho}{ }^{\sigma}=0 .
$$

The curvature tensor $\tilde{R}_{\mu v \rho}{ }^{\sigma}$ has 26 independent components, twenty of which are possessed by $R_{\mu \nu \rho} \sigma$ and six by the conformally invariant field strength $H_{\mu \nu} \equiv \partial_{\mu} S_{v}-\partial_{\nu} S_{\mu}$.

From $\tilde{R}_{\mu v \rho} \sigma$ it is possible to define a conformally invariant Ricci tensor:

$$
\begin{aligned}
\tilde{R}_{\mu v} & \equiv \tilde{R}_{\mu \rho v}{ }^{\rho} \\
& =R_{\mu v}+f\left(-2 \nabla_{\mu} S_{v}-H_{\mu v}-g_{\mu v} \nabla_{\alpha} S^{\alpha}\right)+2 f^{2}\left(S_{\mu} S_{v}-g_{\mu v} S_{\alpha} S^{\alpha}\right) .
\end{aligned}
$$

Let us note that

$$
\tilde{R}_{[\mu v]} \equiv \tilde{R}_{\mu v}-\tilde{R}_{v \mu}=-4 f H_{\mu v} .
$$

Similarly, one can define a conformally not invariant but covariant scalar curvature:

$$
\tilde{R} \equiv g^{\mu v} \tilde{R}_{\mu v}=R-6 f \nabla_{\mu} S^{\mu}-6 f^{2} S_{\mu} S^{\mu} .
$$

We find that under the Weyl transformation (‥J), $\tilde{R} \rightarrow \tilde{R}^{\prime}=e^{-2 \Lambda(x)} \tilde{R}$ while $\tilde{\Gamma}_{\mu v}^{\lambda}, \tilde{R}_{\mu v \rho}{ }^{\sigma}$ and $\tilde{R}_{\mu v}$ are all invariant.

Finally, we wish to write out a generalization of the Gauss-Bonnet topological invariant in the Weyl geometry which can be described as [ए]2]

$$
\begin{aligned}
I_{G B} & \equiv \int d^{4} x \sqrt{-g} \varepsilon^{\mu v \rho \sigma} \varepsilon_{\alpha \beta \gamma \delta} \tilde{R}_{\mu v}^{\alpha \beta} \tilde{R}_{\rho \sigma}^{\gamma \delta} \\
& =-2 \int d^{4} x \sqrt{-g}\left(\tilde{R}_{\mu v \rho \sigma} \tilde{R}^{\rho \sigma \mu \nu}-4 \tilde{R}_{\mu v} \tilde{R}^{v \mu}+\tilde{R}^{2}-12 f^{2} H_{\mu v} H^{\mu v}\right) \\
& =-2 \int d^{4} x \sqrt{-g}\left(R_{\mu v \rho \sigma} R^{\mu v \rho \sigma}-4 R_{\mu v} R^{\mu v}+R^{2}\right) .
\end{aligned}
$$




\section{Weyl invariant quadratic gravity}

In this section, we would like to look for a gravitational theory with the SM based on the Weyl geometry outlined in the previous section. It is of interest to notice that if only the metric tensor is allowed to use for the construction of a gravitational action, the action invariant under the Weyl transformation is restricted to be of form of quadratic gravity, but not be of the EinsteinHilbert type. Using the topological invariant (2.13), up to the kinetic term for the Weyl gauge field, one can write out a general action of quadratic gravity, which is invariant under the Weyl transformation, as follows:

$$
S_{Q G}=\int d^{4} x \sqrt{-g}\left[-\frac{1}{2 \xi^{2}} \tilde{C}_{\mu v \rho \sigma} \tilde{C}^{\mu v \rho \sigma}+\frac{\lambda}{4 !} \tilde{R}^{2}\right] \equiv \int d^{4} x \sqrt{-g} \mathscr{L}_{Q G},
$$

where $\xi$ and $\lambda$ are coupling constants. Moreover, a generalization of the conformal tensor, $\tilde{C}_{\mu v \rho \sigma}$, in the Weyl geometry is defined as in $C_{\mu \nu \rho \sigma}$ in the Riemann geometry:

$$
\begin{aligned}
\tilde{C}_{\mu v \rho \sigma} & \equiv \tilde{R}_{\mu v \rho \sigma}-\frac{1}{2}\left(g_{\mu \rho} \tilde{R}_{v \sigma}+g_{v \sigma} \tilde{R}_{\mu \rho}-g_{\mu \sigma} \tilde{R}_{v \rho}-g_{v \rho} \tilde{R}_{\mu \sigma}\right)+\frac{1}{6}\left(g_{\mu \rho} g_{v \sigma}-g_{\mu \sigma} g_{v \rho}\right) \tilde{R} \\
& =C_{\mu v \rho \sigma}+f\left[-g_{\rho \sigma} H_{\mu v}+\frac{1}{2}\left(g_{\mu \rho} H_{v \sigma}+g_{v \sigma} H_{\mu \rho}-g_{\mu \sigma} H_{v \rho}-g_{v \rho} H_{\mu \sigma}\right)\right] .
\end{aligned}
$$

This conformal tensor in the Weyl geometry has the following properties:

$$
\tilde{C}_{\mu v \rho \sigma}=-\tilde{C}_{v \mu \rho \sigma}, \quad \tilde{C}_{\mu v \rho}{ }^{v}=0, \quad \tilde{C}_{\mu v \rho}{ }^{\rho}=-4 f H_{\mu v} .
$$

Next, by introducing a scalar field $\phi$ and using the classical equivalence, let us rewrite $\tilde{R}^{2}$ in the action (B.D) in the form of the scalar-tensor gravity plus $\lambda \phi^{4}$ interaction [ㅍ3] whose Lagrangian density takes the form

$$
\begin{aligned}
\frac{1}{\sqrt{-g}} \mathscr{L}_{Q G} & =-\frac{1}{2 \xi^{2}} \tilde{C}_{\mu v \rho \sigma} \tilde{C}^{\mu v \rho \sigma}+\frac{\lambda}{12} \phi^{2} \tilde{R}-\frac{\lambda}{4 !} \phi^{4} \\
& =-\frac{1}{2 \xi^{2}} \tilde{C}_{\mu v \rho \sigma} \tilde{C}^{\mu v \rho \sigma}+\frac{1}{12} \phi^{2} \tilde{R}-\frac{\lambda_{\phi}}{4 !} \phi^{4} \\
& =-\frac{1}{2 \xi^{2}} C_{\mu v \rho \sigma} C^{\mu v \rho \sigma}+\frac{1}{12} \phi^{2} R-\frac{\lambda_{\phi}}{4 !} \phi^{4}-\frac{3 f^{2}}{\xi^{2}} H_{\mu v}^{2} \\
& -\frac{1}{2} \phi^{2}\left(f \nabla_{\mu} S^{\mu}+f^{2} S_{\mu} S^{\mu}\right),
\end{aligned}
$$

where in the second equality we have redefined $\sqrt{\lambda} \phi \rightarrow \phi$ and set $\lambda=\frac{1}{\lambda_{\phi}}$. Here it is straightforward to write down a standard model (SM) or physics beyond the standard model (BSM) action which is invariant under the Weyl transformation, but we will omit to tough on it in this article and present the detail in a separate publication. ${ }^{3}$

\section{Emergence of Planck scale}

At low energies, general relativity (GR) describes a lot of gravitational and astrophysical phenomena neatly, so the Weyl invariant Lagrangian density (B.4) of quadratic gravity should be reduced to that of GR at low energies. To do that, we need to break the Weyl symmetry at any rate

\footnotetext{
${ }^{3}$ Some related models on the basis of the Weyl geometry have been made in Refs. [표, 매, 미].
} 
by some method. The standard procedure done so far is to take a gauge condition for the Weyl transformation such that $\phi=\phi_{0}$ where $\phi_{0}$ is a certain constant [0, [1], [13]. However, $\phi_{0}$ is a free parameter which is not fixed by theory in itself so it is not clear why we choose a specific value $\phi_{0} \sim M_{P l}$ where $M_{P l}$ is the Planck mass scale defined as $M_{P l}=\frac{1}{\sqrt{8 \pi G}}=2.44 \times 10^{18} \mathrm{GeV}$ with $G$ being the Newton constant. We wish to construct a theory where the scalar field $\phi$ acquires a vacuum expectation value (VEV) as a result of instabilities in the full quantum theory including quantum corrections from gravity. Technically speaking, what we expect is that after quantum corrections are included the effective potential has a form favoring the specific VEV, $\langle\phi\rangle \sim M_{P l}$.

To this aim, let us first expand the scalar field and the metric around a classical field $\phi_{c}$ and a flat Minkowski metric $\eta_{\mu \nu}$ like [四]

$$
\phi=\phi_{c}+\varphi, \quad g_{\mu v}=\eta_{\mu v}+\xi h_{\mu v}
$$

where we take $\phi_{c}$ to be a constant since we are interested in the effective potential depending on the constant $\phi_{c}$. Next, since we wish to calculate the one-loop effective potential, we will derive only quadratic terms in quantum fields from the classical Lagrangian density (B.4). Then, up to surface terms the Lagrangian density corresponding to the conformal tensor squared takes the form

$$
\mathscr{L}_{C} \equiv-\frac{1}{2 \xi^{2}} \sqrt{-g} C_{\mu v \rho \sigma} C^{\mu v \rho \sigma}=-\frac{1}{4} h^{\mu v} P_{\mu v, \rho \sigma}^{(2)} \square^{2} h^{\rho \sigma},
$$

where $P_{\mu v, \rho \sigma}^{(2)}$ is the projection operator for spin-2 modes ${ }^{4}$ and $\square \equiv \eta^{\mu v} \partial_{\mu} \partial_{v}$. In a similar manner, the Lagrangian density corresponding to the scalar-tensor gravity in Eq. (B.4) reads

$$
\begin{aligned}
\mathscr{L}_{S T} & \equiv \sqrt{-g} \frac{1}{12} \phi^{2} R \\
& =\frac{1}{48} \xi^{2} \phi_{c}^{2} h^{\mu v}\left(P_{\mu v, \rho \sigma}^{(2)}-2 P_{\mu v, \rho \sigma}^{(0, s)}\right) \square h^{\rho \sigma}-\frac{1}{6} \xi \phi_{c} \varphi\left(\eta_{\mu v}-\frac{1}{\square} \partial_{\mu} \partial_{v}\right) \square h^{\mu \nu} .
\end{aligned}
$$

The remaining Lagrangian density can be evaluated in a similar way and consequently all the quadratic terms in (B.4) are summarized to be

$$
\begin{aligned}
\mathscr{L}_{Q G} & =\frac{1}{4} h^{\mu v}\left[\left(-\square+\frac{1}{12} \xi^{2} \phi_{c}^{2}\right) P_{\mu v, \rho \sigma}^{(2)}-\frac{1}{6} \xi^{2} \phi_{c}^{2} P_{\mu v, \rho \sigma}^{(0, s)}\right] \square h^{\rho \sigma} \\
& -\frac{1}{6} \xi \phi_{c} \varphi\left(\eta_{\mu v}-\frac{1}{\square} \partial_{\mu} \partial_{\nu}\right) \square h^{\mu \nu}-\frac{\lambda_{\phi}}{4} \phi_{c}^{2} \varphi^{2}-\frac{\lambda_{\phi}}{12} \xi \phi_{c}^{3} h \varphi \\
& -\frac{3 f^{2}}{\xi^{2}} H_{\mu \nu}^{\prime 2}-\frac{1}{2} f^{2} \phi_{c}^{2} S_{\mu}^{\prime} S^{\prime \mu}-\frac{1}{2} \varphi \square \varphi,
\end{aligned}
$$

where we have set $S_{\mu}^{\prime}=S_{\mu}-\frac{1}{f \phi_{c}} \partial_{\mu} \varphi$ and $H_{\mu v}^{\prime}=\partial_{\mu} S_{v}^{\prime}-\partial_{v} S_{\mu}^{\prime}$.

At this stage, let us set up the gauge-fixing conditions. For diffeomorphisms, we adopt a gauge condition

$$
\chi_{\mu} \equiv \xi \partial^{v}\left(h_{\mu v}-\frac{1}{4} \eta_{\mu v} h\right)=0,
$$

\footnotetext{
${ }^{4}$ We follow the definition of projection operators in [미, 따].
} 
which is invariant under the conformal transformation. We find that the Lagrangian density for this gauge condition and its corresponding FP ghost term is given by

$$
\mathscr{L}_{G F+F P}=-\frac{1}{2 \alpha} \chi_{\mu} Y^{\mu v} \chi_{v}-\bar{c}_{\mu} Y^{\mu v}\left(\square c_{v}+\frac{1}{2} \partial_{v} \partial_{\rho} c^{\rho}\right),
$$

where $\alpha$ is a gauge parameter. Let us recall that in case of the higher derivative gravity [एव, [0]], it is convenient to work with a more general gauge-fixing term $\mathscr{L}_{G F}=-\frac{1}{2 \alpha} \chi_{\mu} Y^{\mu v} \chi_{v}$ where $Y^{\mu v}$ is the weight function involving derivatives and gauge parameters [2], [22]. For instance, a suitable choice is $Y^{\mu v}=\eta^{\mu v} \square+c \partial^{\mu} \partial^{v}$ ( $c$ is a constant) and in calculating the propagators, the constants $c$ and $\alpha$ are fixed to be an appropriate value for simplifying the expressions. Next, we fix the gauge freedom corresponding to the Weyl transformation by a gauge condition

$$
h \equiv \eta^{\mu v} h_{\mu v}=0 .
$$

Since this gauge fixing condition and its Weyl transformation contain no derivatives, one can neglect the corresponding FP ghost term in the one-loop approximation.

Consequently, we can obtain a quantum Lagrangian density involving only quadratic terms:

$$
\begin{aligned}
\mathscr{L}_{Q G} & =\frac{1}{4} h^{\mu v}\left(-\square+\frac{1}{12} \xi^{2} \phi_{c}^{2}\right) P_{\mu v, \rho \sigma}^{(2)} \square h^{\rho \sigma}-\frac{1}{72} \xi^{2} \phi_{c}^{2} \partial_{\mu} \partial_{v} h^{\mu v} \frac{1}{\square} \partial_{\rho} \partial_{\sigma} h^{\rho \sigma} \\
& +\frac{1}{6} \xi \phi_{c} \varphi \partial_{\mu} \partial_{v} h^{\mu v}-\frac{1}{2} \varphi\left(\square+\frac{1}{2} \lambda_{\phi} \phi_{c}^{2}\right) \varphi-\frac{1}{4} \hat{H}_{\mu v}^{2}-\frac{1}{2} \frac{\xi^{2} \phi_{c}^{2}}{12} \hat{S}_{\mu} \hat{S}^{\mu} \\
& -\frac{1}{2 \alpha} \chi_{\mu} Y^{\mu v} \chi_{v}-\bar{c}_{\mu} Y^{\mu v}\left(\square c_{v}+\frac{1}{2} \partial_{v} \partial_{\rho} c^{\rho}\right),
\end{aligned}
$$

where we have introduced $\hat{S}_{\mu} \equiv \frac{2 \sqrt{3} f}{\xi} S_{\mu}^{\prime}$, defined $\hat{H}_{\mu \nu} \equiv \partial_{\mu} \hat{S}_{v}-\partial_{v} \hat{S}_{\mu}$ and used the gauge condition (4.]). Moreover, in order to eliminate the mixed term involving $\varphi$ and $h_{\mu v}$, let us introduce a new field $\hat{\varphi}$ instead of $\varphi$, which is defined as

$$
\hat{\varphi} \equiv \varphi-\frac{1}{6} \xi \phi_{c}\left(\square+\frac{1}{2} \lambda_{\phi} \phi_{c}^{2}\right)^{-1} \partial_{\mu} \partial_{v} h^{\mu \nu}
$$

Then, the Lagrangian density (4.8) can be rewritten as

$$
\begin{aligned}
\mathscr{L}_{Q G} & =\frac{1}{4} h^{\mu v}\left(-\square+\frac{1}{12} \xi^{2} \phi_{c}^{2}\right) P_{\mu v, \rho \sigma}^{(2)} \square h^{\rho \sigma}-\frac{1}{4} \hat{H}_{\mu v}^{2}-\frac{1}{2} \frac{\xi^{2} \phi_{c}^{2}}{12} \hat{S}_{\mu} \hat{S}^{\mu} \\
& -\frac{1}{2} \hat{\varphi}\left(\square+\frac{1}{2} \lambda_{\phi} \phi_{c}^{2}\right) \hat{\varphi}-\frac{1}{144} \lambda_{\phi} \xi^{2} \phi_{c}^{4} \partial_{\mu} \partial_{v} h^{\mu v} \frac{1}{\square\left(\square+\frac{1}{2} \lambda_{\phi} \phi_{c}^{2}\right)} \partial_{\rho} \partial_{\sigma} h^{\rho \sigma} \\
& -\frac{1}{2 \alpha} \chi_{\mu} Y^{\mu v} \chi_{v}-\bar{c}_{\mu} Y^{\mu v}\left(\square c_{v}+\frac{1}{2} \partial_{v} \partial_{\rho} c^{\rho}\right) .
\end{aligned}
$$

Based on this quantum Lagrangian density (4.]J), we can evaluate the one-loop effective action by integrating out quantum fluctuations. Then, up to a classical potential, the effective action $\Gamma\left[\phi_{c}\right]$ reads

$$
\Gamma\left[\phi_{c}\right]=i \frac{5+3}{2} \log \operatorname{det}\left(-\square+\frac{1}{12} \xi^{2} \phi_{c}^{2}\right)
$$


Here some remarks are in order. First, in this expression, the factors 5 and 3 come from the fact that a massive spin-2 state and a massive spin-1 Weyl gauge field possess five and three physical degrees of freedom, respectively. In order to see the pole structure of the massive Weyl gauge field $\hat{S}_{\mu}$ more easily, it might be useful to introduce a scalar field $\pi$ to ensure the gauge symmetry. Indeed, redefining $\hat{S}_{\mu} \rightarrow \hat{S}_{\mu}+\partial_{\mu} \pi$ and then integrating over $\pi$ leads to the following gauge invariant expression:

$$
\begin{aligned}
\mathscr{L}_{S} & \equiv-\frac{1}{4} \hat{H}_{\mu \nu}^{2}-\frac{1}{2} \frac{\xi^{2} \phi_{c}^{2}}{12}\left(\hat{S}_{\mu}+\partial_{\mu} \pi\right)^{2} \\
& \rightarrow \frac{1}{4} \hat{H}_{\mu \nu} \frac{-\square+\frac{\xi^{2} \phi_{c}^{2}}{12}}{\square} \hat{H}^{\mu v} .
\end{aligned}
$$

Taking the Lorenz gauge condition $\partial_{\mu} \hat{S}^{\mu}=0$, the Lagrangian density becomes

$$
\mathscr{L}_{S}=-\frac{1}{2} \hat{S}_{\mu}\left(-\square+\frac{\xi^{2} \phi_{c}^{2}}{12}\right) \hat{S}^{\mu}
$$

This form clearly shows that the massive gauge field at hand has the mass squared $\frac{\xi^{2} \phi_{c}^{2}}{12}$, but one disadvantage is that not three but two degrees of freedom has such a property. The remaining one degree of freedom is contained in the longitudinal mode which is integrated over in the above derivation. Finally, it is valuable to note that the determinant $\operatorname{det}\left(\square+\frac{1}{2} \lambda_{\phi} \phi_{c}^{2}\right)$ coming from the scalar field $\hat{\varphi}$ is precisely cancelled by that from the metric tensor, which implies that "dilaton" $\hat{\varphi}$ is no longer a dynamical mode and is absorbed into the logitudinal mode of the Weyl gauge field, thereby the massless gauge field becoming a massive one with mass squared equal to $\frac{\xi^{2} \phi_{c}^{2}}{12}$. Also note that we have ignored the part of the effective action which is independent of $\phi_{c}$ since it never gives us the effective potential for $\phi_{c}$.

To calculate $\Gamma\left[\phi_{c}\right]$, we will proceed step by step: First, let us note that $\Gamma\left[\phi_{c}\right]$ can be rewritten as follows:

$$
\begin{aligned}
\Gamma\left[\phi_{c}\right] & =4 i \operatorname{Tr} \log \left(-\square+\frac{1}{12} \xi^{2} \phi_{c}^{2}\right) \\
& =4 i \int d^{4} x\left\langle x\left|\log \left(-\square+\frac{1}{12} \xi^{2} \phi_{c}^{2}\right)\right| x\right\rangle \\
& =4 i \int d^{4} x \int \frac{d^{4} k}{(2 \pi)^{4}}\left\langle x\left|\log \left(-\square+\frac{1}{12} \xi^{2} \phi_{c}^{2}\right)\right| k\right\rangle\langle k \mid x\rangle \\
& =4 i(V T) \int \frac{d^{4} k}{(2 \pi)^{4}} \log \left(k^{2}+\frac{1}{12} \xi^{2} \phi_{c}^{2}\right) \\
& =4(V T) \frac{\Gamma\left(-\frac{d}{2}\right)}{(4 \pi)^{\frac{d}{2}}}\left(\frac{1}{12} \xi^{2} \phi_{c}^{2}\right)^{\frac{d}{2}},
\end{aligned}
$$

where $(V T)$ denotes the space-time volume and in the last equality we have used the Wick rotation and the dimensional regularization.

Next, let us evaluate $\Gamma\left[\phi_{c}\right]$ in terms of the modified minimal subtraction scheme. In this scheme, the $\frac{1}{\varepsilon}$ poles (where $\varepsilon \equiv 4-d$ ) together with the Euler-Mascheroni constant $\gamma$ and $\log (4 \pi)$ 
are subtracted and then replaced with $\log M^{2}$ where $M$ is an arbitrary mass parameter which is introduced to make the final equation dimensionally correct [23] . By subtracting the $\frac{1}{\varepsilon}$ pole, (4.14) is reduced to the form

$$
\begin{aligned}
-\frac{1}{V T} \Gamma\left[\phi_{c}\right] & =-4 \frac{\Gamma\left(2-\frac{d}{2}\right)}{\frac{d}{2}\left(\frac{d}{2}-1\right)} \frac{1}{(4 \pi)^{\frac{d}{2}}}\left(\frac{1}{12} \xi^{2} \phi_{c}^{2}\right)^{\frac{d}{2}} \\
& =-\frac{4}{2(4 \pi)^{2}}\left(\frac{1}{12} \xi^{2} \phi_{c}^{2}\right)^{2}\left[\frac{2}{\varepsilon}-\gamma+\log (4 \pi)-\log \left(\frac{1}{12} \xi^{2} \phi_{c}^{2}\right)+\frac{3}{2}\right] \\
& \rightarrow \frac{2}{(4 \pi)^{2}}\left(\frac{1}{12} \xi^{2} \phi_{c}^{2}\right)^{2}\left[\log \left(\frac{\xi^{2} \phi_{c}^{2}}{12 M^{2}}\right)-\frac{3}{2}\right]
\end{aligned}
$$

Then, the one-loop effective potential will be of form ${ }^{5}$

$$
V_{e f f}^{(1)}\left(\phi_{c}\right)=c_{1}+c_{2} \phi_{c}^{2}+\frac{1}{1152 \pi^{2}} \xi^{4} \phi_{c}^{4} \log \left(\frac{\phi_{c}^{2}}{c_{3}}\right)
$$

where $c_{i}(i=1,2,3)$ are constants to be determined by the renormalization conditions:

$$
\left.V_{e f f}^{(1)}\right|_{\phi_{c}=0}=\left.\frac{d^{2} V_{e f f}^{(1)}}{d \phi_{c}^{2}}\right|_{\phi_{c}=0}=\left.\frac{d^{4} V_{e f f}^{(1)}}{d \phi_{c}^{4}}\right|_{\phi_{c}=\mu}=0,
$$

where $\mu$ is the renormalization mass. As a result, we have the one-loop effective potential

$$
V_{e f f}^{(1)}\left(\phi_{c}\right)=\frac{1}{1152 \pi^{2}} \xi^{4} \phi_{c}^{4}\left(\log \frac{\phi_{c}^{2}}{\mu^{2}}-\frac{25}{6}\right) .
$$

Finally, by adding the classical potential we can arrive at the effective potential in the one-loop approximation

$$
V_{e f f}\left(\phi_{c}\right)=\frac{\lambda_{\phi}}{4 !} \phi_{c}^{4}+\frac{1}{1152 \pi^{2}} \xi^{4} \phi_{c}^{4}\left(\log \frac{\phi_{c}^{2}}{\mu^{2}}-\frac{25}{6}\right)
$$

It is easy to see that this effective potential has a minimum at $\phi_{c}=\langle\phi\rangle$ away from the origin where the effective potential, $V_{\text {eff }}(\langle\phi\rangle)$, is negative. Since the renormalization mass $\mu$ is arbitrary, we will choose it to be the actual location of the minimum, $\mu=\langle\phi\rangle[$ [] $]$ :

$$
V_{e f f}\left(\phi_{c}\right)=\frac{\lambda_{\phi}}{4 !} \phi_{c}^{4}+\frac{1}{1152 \pi^{2}} \xi^{4} \phi_{c}^{4}\left(\log \frac{\phi_{c}^{2}}{\langle\phi\rangle^{2}}-\frac{25}{6}\right) .
$$

Since $\phi_{c}=\langle\phi\rangle$ is defined to be the minimum of $V_{e f f}$, we deduce

$$
\begin{aligned}
0 & =\left.\frac{d V_{e f f}}{d \phi_{c}}\right|_{\phi_{c}=\langle\phi\rangle} \\
& =\left(\frac{\lambda_{\phi}}{6}-\frac{11}{864 \pi^{2}} \xi^{4}\right)\langle\phi\rangle^{3},
\end{aligned}
$$

\footnotetext{
${ }^{5}$ At first sight, the existence of the $c_{2} \phi_{c}^{2}$ might appear to be strange, but this term in fact emerges in the cutoff regularization. Note that the only logarithmically divergent term, but not quadratic divergent one, arises in the dimensional regularization.
} 
or equivalently,

$$
\lambda_{\phi}=\frac{11}{144 \pi^{2}} \xi^{4} .
$$

This relation is similar to $\lambda=\frac{33}{8 \pi^{2}} e^{4}$ in case of the scalar QED in Ref. [B]], so as in that paper, the perturbation theory holds for very small $\xi$ as well.

The substitution of Eq. (4.22) into $V_{\text {eff }}$ in (4.20) leads to

$$
V_{e f f}\left(\phi_{c}\right)=\frac{1}{1152 \pi^{2}} \xi^{4} \phi_{c}^{4}\left(\log \frac{\phi_{c}^{2}}{\langle\phi\rangle^{2}}-\frac{1}{2}\right) .
$$

Thus, the effective potential is now parametrized in terms of $\xi$ and $\langle\phi\rangle$ instead of $\xi$ and $\lambda_{\phi}$; it is nothing but dimensionaltransmutation, i.e., a dimensionless coupling constant $\lambda_{\phi}$ is traded for a dimensional quantity $\langle\phi\rangle$ via symmetry breakdown of the local Weyl symmetry.

Hence, from the classical Lagrangian density (B.4) of quadratic gravity, via dimensional transmutation, the Einstein-Hilbert term for GR is induced in such a way that the Planck mass $M_{P l}$ is given by

$$
M_{P l}^{2}=\frac{1}{6}\langle\phi\rangle^{2} .
$$

At the same time, the Weyl gauge field becomes massive by 'eating' the dilaton $\varphi$ whose magnitude of mass is given

$$
m_{S}^{2}=\frac{1}{12} \xi^{2}\langle\phi\rangle^{2}=\frac{1}{2} \xi^{2} M_{P l}^{2} .
$$

As long as the perturbation theory is concerned, the coupling constant $\xi$ must take a small value, $\xi \ll 1$. At the low energy region satisfying $E \ll m_{S}$, we can integrate over the massive Weyl gauge field, and consequently not only we would have GR with the SM but also the second clock effect has no physical effects at low energies.

\section{Emergence of electroweak scales}

Though we have ignored the existence of the SM and limited ourselves to the gravitational sector thus far, it is straightforward to incorporate the SM or the BSM in the present formulation by replacing the gauge covariant derivative with the Weyl covariant one. As for the Higgs sector, since the (tachyonic) Higgs mass term breaks the scale symmetry, after the symmetry breakdown of the local Weyl symmetry by the Coleman-Weinberg mechanism, the Higgs potential must be replaced by a potential

$$
V_{e f f}(\phi, H)=\frac{1}{1152 \pi^{2}} \xi^{4} \phi^{4}\left(\log \frac{\phi^{2}}{\langle\phi\rangle^{2}}-\frac{1}{2}\right)+\lambda_{H \phi}\left(H^{\dagger} H\right) \phi^{2}+\frac{\lambda_{H}}{2}\left(H^{\dagger} H\right)^{2} .
$$

Inserting the minimum $\phi=\langle\phi\rangle$ to Eq. (5. لل and completing the square, the effective potential reduces to

$$
V_{e f f}(\langle\phi\rangle, H)=\frac{\lambda_{H}}{2}\left(H^{\dagger} H+\frac{\lambda_{H \phi}}{\lambda_{H}}\langle\phi\rangle^{2}\right)^{2}-\frac{1}{2}\left(\frac{\lambda_{H \phi}^{2}}{\lambda_{H}}+\frac{1}{1152 \pi^{2}} \xi^{4}\right)\langle\phi\rangle^{4} .
$$


Owing to $\lambda_{H}>0$, this potential has a minimum at $H^{\dagger} H=-\frac{\lambda_{H \phi}}{\lambda_{H}}\langle\phi\rangle^{2}$. Taking the unitary gauge $H^{T}=\frac{1}{\sqrt{2}}(0, v+h)$, this fact implies that the squares of the VEV $v$ and the Higgs mass $m_{h}$ are given by

$$
v^{2}=\frac{2\left|\lambda_{H \phi}\right|}{\lambda_{H}}\langle\phi\rangle^{2}, \quad m_{h}^{2}=\lambda_{H} v^{2}
$$

Using Eqs. (4.24) and (5.3)), the magnitude of the coupling constant $\lambda_{H \phi}$ reads

$$
\left|\lambda_{H \phi}\right|=\frac{1}{12}\left(\frac{m_{h}}{M_{p l}}\right)^{2} \sim \mathscr{O}\left(10^{-33}\right) .
$$

This relation $\left|\lambda_{H \phi}\right| \ll 1$ implies that the Higgs sector (or the SM sector) almost decouples from the gravitational sector but the mixed term $\lambda_{H \phi}\left(H^{\dagger} H\right) \phi^{2}$ in the potential (D.J) plays an important role in the sense that the VEV, $\langle\phi\rangle$, obtained by the Coleman-Weinberg mechanism around the Planck mass scale, is transmitted to the SM sector, thereby generating the Higgs mass term.

\section{Conclusions}

Shortly after Einstein constructed general relativity (GR) in 1915, Weyl has advocated a generalization in that the very notion of length becomes path-dependent [ [⿴囗十, []]. In Weyl's theory, even if the lightcones retain the fundamental role as in GR, there is no absolute meaning of scales for space-time, so the metric is defined only up to proportionality. It is this property that we have a scale symmetry prohibiting the appearance of any dimensionful parameters and coupling constants in the Weyl theory. The main complaint against the Weyl's idea is that it inevitably leads to the so-called "second clock effect": The rate where any clock measures would depend on its history [四]. Since the second clock effect has not been observed by experiments, the Weyl theory might make no sense as a classical theory.

However, viewed as a quantum field theory, the Weyl theory is a physically consistent and interesting theory and provides us with a natural playground for constructing conformally invariant quantum field theories as shown in this article. ${ }^{6}$ Requiring the invariance under Weyl transformation is so strong that only quadratic curvature terms are allowed to exist in a classical action, which should be contrasted with the situation of GR where any number of curvature terms could be in principle the candidate of a classical action only if we require the action to be invariant under diffeomorphisms.

Of course, we have a serious problem to be solved in future; the problem of unitarity. The lack of perturbative unitarity is a common problem in the higher derivative gravity like the Weyl theory. However, it is expected that the Weyl gravity is asymptotically free, and the issue of the perturbative unitarity is closely relevant to infrared dynamics of asymptotic fields, so this problem becomes quite nontrivial. Provided that we can confine the ghosts in the Weyl theory like in QCD, we would be free of the perturbative unitarity.

\section{Acknowledgements}

\footnotetext{
${ }^{6}$ We have already contructed the other scale invariant gravitational models [24, [25, [26].
} 
After submitting the paper [四], we were informed by F. F. Faria of the reference [27] where the effective potential of the conformal gravity in the Riemann geometry has been evaluated by using the DeWitt-Schwinger method. We are grateful to F. F. Faria for this useful information. The present work was supported by JSPS KAKENHI Grant Number 16K05327.

\section{References}

[1] I. Oda, "Planck and Electroweak Scales Emerging from Conformal Gravity", arXiv:1806.03420 [hep-th].

[2] C. W. Misner, K. S. Thorne and J. A. Wheeler, "Gravitation", W H Freeman and Co (Sd), 1973.

[3] S. R. Coleman and E. J. Weinberg, "Radiative Corrections as the Origin of Spontaneous Symmetry Breaking", Phys. Rev. D 7 (1973) 1888.

[4] H. Weyl, "Gravitation und Elekrizität", Sitzungsberichte der Königlich Preußischen Akademie der Wissenschaften zu Berlin, 1918, pp. 465-480.

[5] E. Scholz, "The Unexpected Resurgence of Weyl Geometry in late 20th-Century Physics", Einstein Stud. 14 (2018) 261.

[6] R. Penrose, "The Road to Reality", Vintage Books, New York, 2004.

[7] I. Oda, "Higgs Potential from Wick Rotation in Conformal BSM", arXiv:1810.02478 [hep-th].

[8] T. Fulton, F. Rohrlich and L. Witten, "Conformal Invariance in Physics", Rev. Mod. Phys. 34 (1962) 442.

[9] L. Smolin, "Towards a Theory of Spacetime Structure at Very Short Distances", Nucl. Phys. B 160 (1979) 253.

[10] M. de Cesare, J. W. Moffat and M. Sakellariadou, "Local Conformal Symmetry in Non-Riemannian Geometry and the Origin of Physical Scales", Eur. Phys. J. C 77 (2017) 605.

[11] R. M. Wald, "General Relativity", University of Chicago Press, Chicago, 1984.

[12] W. Drechsler and H. Tann, , "Broken Weyl Invariance and the Origin of Mass", Found. Phys. 29 (1999) 1023.

[13] D. M. Ghilencea, "Spontaneous Breaking of Weyl Quadratic Gravity to Einstein Action and Higgs Potential", arXiv:1812.08613 [hep-th].

[14] H. Cheng, "Possible Existence of Weyl's Vector Meson", Phys. Rev. Lett. 61 (1988) 2182.

[15] H. Nishino and S. Rajpoot, "Broken Scale Invariance in the Standard Model", arXiv: 0403039 [hep-th].

[16] H. Nishino and S. Rajpoot, "Implication of Compensator Field and Local Scale Invariance in the Standard Model", Phys. Rev. D 79 (2009) 125025.

[17] M. Nakasone and I. Oda, "On Unitarity of Massive Gravity in Three Dimensions", Prog. Theor. Phys. 121 (2009) 1389.

[18] M. Nakasone and I. Oda, "Massive Gravity with Mass Term in Three Dimensions", Phys. Rev. D 79 (2009) 104012.

[19] K. S. Stelle, "Renormalization of Higher Derivative Quantum Gravity", Phys. Rev. D 16 (1977) 953. 
[20] J. Julve and M. Tonin, "Quantum Gravity with Higher Derivative Terms", Nuovo Cim. B 46 (1978) 137.

[21] E. S. Fradkin and A. A. Tseytlin, "Renormalizable Asymptotically Free Quantum Theory of Gravity", Nucl. Phys. B 201 (1982) 469.

[22] I. L. Buchbinder, S. D. Odintsov and I. L. Shapiro, "Effective Actions in Quantum Gravity", IOP Publishing Ltd, London, 1992.

[23] M. E. Peskin and D. V. Schroeder, "An Introduction to Quantum Field Theory", Westview Press, Boulder, 1995.

[24] I. Oda, "Classically Scale-invariant B-L Model and Dilaton Gravity", Phys. Rev. D 87 (2013) 065025.

[25] I. Oda, "Classically Scale-invariant B-L Model and Conformal Gravity", Phys. Lett. B 724 (2013) 160 .

[26] I. Oda, "Higgs Mechanism in Scale-Invariant Gravity", Adv. Studies in Theor. Phys. 8 (2014) 215.

[27] N. Matsuo, "Einstein Gravity as Spontaneously Broken Weyl Gravity", Gen. Rel. Grav. 22 (1990) 561. 